KENTRON

EVUE PLURIDISCIPUINAIRE

DU MONDE ANTIQUE

\section{Kentron}

Revue pluridisciplinaire du monde antique

23 | 2007

La démesure (fin)

\title{
Emendativncvlae Malaterrenae II
}

\section{Olivier Desbordes}

\section{OpenEdition}

\section{Journals}

Édition électronique

URL : http://journals.openedition.org/kentron/1747

DOI : 10.4000/kentron. 1747

ISSN : 2264-1459

\section{Éditeur}

Presses universitaires de Caen

\section{Édition imprimée}

Date de publication : 31 décembre 2007

Pagination : 55-77

ISBN : 978-2-84133-321-9

ISSN : 0765-0590

Référence électronique

Olivier Desbordes, «Emendativncvlae Malaterrenae II », Kentron [En ligne], 23 | 2007, mis en ligne le 16 mars 2018, consulté le 20 novembre 2020. URL : http://journals.openedition.org/kentron/1747 ; DOI : https://doi.org/10.4000/kentron.1747

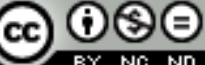

Kentron is licensed under a Creative Commons Attribution-NonCommercial-NoDerivatives 3.0 International License. 


\section{EMENDATIVNCVLAE MALATERRENAE II $^{1}$}

\section{Petro Bouet septuagenario}

II Praef. (p. 29, 2 sq.) Si quaeritur quod, Calabria uel Apulia iam ex parte, non autem ex toto, quantum ad ea quae in eis facta sunt, descriptis, apud Siciliam describendam transimus [...], sciendum est quod eqs.

$\|$ quaeritur $C Z$, edd. : -ratur $B \|$ quod $C Z$ : quare $B \|$ describendam $C$ : -bentes $Z B$, edd. $\|$ transimus $C Z B$, Avenel: -seamus edd. uett. Pontieri $\|$

Si quaeritur donne un sens a priori acceptable, quod ne peut pas ne pas éveiller la défiance : en effet, il ne se joint pas ordinairement avec quaero pour introduire la complétive que ce verbe régit. Quant à l'indicatif transimus, il soulève lui aussi dans ce contexte une difficulté, n'étant pas mieux justifié au point de vue de la syntaxe que la subordination par quod ${ }^{2}$. Ces deux difficultés seront écartées si, derrière le quaeritur de la tradition imprimée, on sait reconnaître le mot que la grammaire réclame, queritur ${ }^{3}$ : quod est usuel après un verbe de sentiment comme queror ${ }^{4}$; et

1. Notre collègue Marie-Agnès Avenel a bien voulu, une fois encore, relire nos observations critiques sur le texte de Malaterra. Il nous est très agréable de lui exprimer à nouveau notre amicale gratitude. La pagination et la linéation que nous indiquons entre parenthèses sont celles de l'édition Pontieri.

2. Supposant que, après quaeritur, quod pouvait être pris dans le sens de cur, l'édition princeps - et tous les éditeurs successifs jusqu'à E. Pontieri inclusivement ne se sont pas prononcés différemment - a conformé la construction aux habitudes de la grammaire et substitué le subjonctif à l'indicatif que sa source manuscrite $(Z)$ lui indiquait, atténuant ainsi l'apparente singularité du tour. Quant au quare de B, ce n'est qu'une de ces fausses corrections qui fourmillent dans ce témoin de faible autorité. On y a d'ailleurs, illogiquement, réformé quaeritur sans retoucher à transimus. En III 25 (p. 72 , 25), quaeritur est construit avec un subjonctif de volonté, sans ut: Ereptum ueniant, cum prece quaeritur (vers asclépiade).

3. Rappelons qu'aucune des sources manuscrites employées pour la constitution du texte n'est antérieure au XIV $\mathrm{X}^{\mathrm{e}}$ siècle et ne distingue $e$ de $a e$, sauf $\mathrm{C}$, qui recourt occasionnellement au $e$ cédillé.

4. Ce quod complétif apparaît encore après un verbe de sentiment en II 45 (p. 53, 13: si certi sunt quod non cogantur ullis iniustis), IV 8 (p. 90, 16 sq. : habebat denique fiduciam in comite [...] quod eam [sc. filiam suam] regi honeste consignaret) et IV 20 (p. 99, 13 sq. : indignatione permotus quod talia facere praesumpserit). On le trouve également après des verbes et locution signifiant «blâmer, reprocher » : cf. I 16 (p. 17, 12 : redarguitur [...] quod talia praesumpserit), III 31 (p. 76, 27 sq. : increpans eum [...] quod tale quid praesumpsisset) et IV 13 (p. 92, 32-34: paterna increpatione submonuerat quod [...] interdixerat). 
l'indicatif, mode de l'affirmation directe, apparaît dans une complétive dont le contenu est posé comme incontestable: à ce moment de son récit, le chroniqueur, respectueux de la chronologie, aborde la conquête de la Sicile par le comte Roger. De toute évidence, dans cette préface construite en manière d'anticipatio, Malaterra entend répondre sur un point précis à l'indignatio de ses détracteurs. L'objection qu'on lui adresse est énoncée dans l'ablatif absolu Calabria uel Apulia iam ex parte, non autem ex toto, quantum ad ea quae in eis facta sunt, descriptis ${ }^{5}$. Deux conceptions ici se font jour : à une démarche purement chronologique qui, privilégiant l'ordre des temps ${ }^{6}$, donne le pas à la suite des événements sur le cadre géographique et passe alternativement, au gré de ces événements, d'une sphère d'opérations déterminées - l'ApulieCalabre - à l'autre - la Sicile - sans souci de l'unité de lieu, s'oppose une conception pour ainsi dire «monographique » qui, consacrant le primat du cadre géographique, inscrit la suite des événements dans un théâtre d'opérations particulier et s'interdit d'en franchir les frontières avant que le récit n'ait épuisé les événements qui s'y sont déroulés. Ainsi, selon ces contradicteurs, la conquête de la Sicile est exclusive de celle de l'Italie du Sud: l'une et l'autre relève d'un récit spécifique. De là ce Si queritur quod... («Si l'on déplore que...»), écho atténué des récriminations évoquées - parfois sur le même mode - dans les deux épîtres dédicatoires. Et l'on redressera de même, p. 3, 17, le quaestio des éditeurs et des traducteurs pour lire, avec E. D’Angelo ${ }^{7}$ : Si [...] de incultiori poetria questio fuerit [...]. La différence de sens qu'entraîne à chaque fois la substitution d'une leçon à l'autre n'est pas de grande conséquence, mais c'est le devoir de la critique de donner - ou retrouver - ce que Malaterra a écrit.

II 5 (p. 31, 10 sq.) Messanenses putantes, quibusdam iam naues ingressis, se illos quasi semipartitos facilius posse occupare, eqs.

\|n naues $Z^{2}$,ed.pr. Pontieri: nauibus $C Z^{1} B$, Avenel $\|$

nauibus, qui a été transmis par les trois manuscrits à notre disposition et dont M.-A. Avenel a respecté l'autorité, n'a probablement qu'une très faible valeur, la construction dative ${ }^{8}$ ''ingredior constituant un hapax dans l'usage grammatical de

5. Trad. littérale : «la Calabre et l'Apulie ayant été traitées pour le moment partiellement et non en totalité pour ce qui est des événements qui s'y sont produits ».

6. Sur l'autorité impérieuse de l'ordre chronologique, qui commande au chroniqueur de passer outre aux réactions vives suscitées chez ses contemporains par l'évocation d'un malheur cuisant, cf. IV 18 (p. 97, 6-12).

7. Cf. D’Angelo 2003, 135. La correction n'est pas motivée. Lo Curto 2002, 20-21, a imprimé quaestio, comme Pontieri, son prototype, mais au rebours de la plupart des traducteurs modernes, il ne fait pas dire à Malaterra ce qu'il ne dit pas : «Si invece mi si rimprovera [...] ». En II praef., l'attaque naturelle de la phrase d'ouverture en a imposé à tous, et, en dépit des incertitudes de la construction, le passif impersonnel quaeritur a été consacré.

8. Nous excluons d'emblée, bien qu'on puisse appuyer cette construction sur quelques cas parallèles - cf. Flobert 1975,373-, qu'ingressis soit un perfectum passif dont quibusdam nauibus serait le constituant 
Malaterra ${ }^{9}$. Ce datif pourrait s'expliquer comme une réminiscence directe ou indirecte de Virgile: en effet, le datif avec ingredior pour indiquer le point où le mouvement aboutit ne se rencontre guère que chez les poètes ${ }^{10}$, et on ne saurait dénier a priori au chroniqueur le droit d'user de tournures poétiques ${ }^{11}$. Mais rien, dans ce passage narratif, ne légitime une telle recherche de style. Plutôt qu'une lectio difficilior, nauibus représente une leçon fautive née pendant la transmission du texte de l'archétype à l'ancêtre dont nos deux classes de manuscrits sont issues: un copiste a pu, à son insu, substituer nauibus à naues sous l'influence immédiate des deux ablatifs qui enserrent le substantif. Le changement de syntaxe ne répond pas à une intention du chroniqueur; il est accidentel, et M.-A. Avenel a laissé échapper ici une irrégularité dont nous sommes redevables à un copiste. Des effets de suggestion de cette nature ont été déjà signalés : cf. Desbordes 2005, 135, ad II $7^{12}$.

sujet. nauibus ingressis ne saurait entrer en parallèle avec nauibus ascensis (III 9 [p. 61, 16]), à quoi on peut faire correspondre naues ascendit (III 11 [p. 63, 23]).

9. Outre II 5 , on relève dix-sept emplois d'ingredior dans la chronique - dont quatre avec nauis (cf. I 37 [p. 24, 13: nauem ingressi] ; II 1 [p. 30, 4: naues suas ingressus]; IV 16 [p. 94, 37 : naues ingredi; p. 95, 11: naues ingressus]) - sans que jamais la construction du verbe varie: le substantif est mis toujours à l'accusatif (castrum: II 28 [p. 39, 17 sq.] ; II 33 [p. 43, 2] ; III 9 [p. 61, 21] ; IV 16 [p. 94, 41] - hortos: II 45 [p. 53,3] - lacum: I praef. [p. 4, 15] - palatium [représenté par un relatif de liaison] : II 24 [p. 37, 17] - urbem: II 24 [p. 37, 10; 37, 16;37, 18] ; IV 16 [p. 94, 41] ; IV 18 [p. 98, 1] ; Sckillacium: I 37 [p. 24, 11] ; Stilum: II 44 [p. 52, 11 (apparat) : reuersus B, Pontieri]). ingredi avec l'accusatif est admis en prose depuis bien longtemps : cf. Kühner et al. 1976, II 1, 267; Saint-Denis 1935, 73.

10. Voir TLL VII 1, 1575, 61: «dat. loci in poesi inde a Verg(ilio)»; cf. Aen. 10, 148 castris ingressus Etruscis. Voir aussi Marouzeau 1962, 210 : «On sait [...] que la construction a été mise à la mode par la poésie : Cicéron ne l'admet que dans des traductions de poètes [...], et c'est Virgile qui en fait définitivement le succès ».

11. Par exemple, l'accusatif après insilio est un poétisme avéré (cf. TLL VII 1, 1910, 67-77 [GonzálezHaba, 1958]); cf. I 13 (p. 15, 1 sq. : castra [...] insiluit); I 26 (p. 21, 3: equum insiliens); IV 2 (p. 86, 18 sq. : dum a comite, qui nauem eius insiluerat [insilierat $\mathrm{C}$ ], minaci ense persequitur). Mais dans ce cas, l'accusatif est employé de manière constante.

12. Le hasard des relectures nous en a donné un nouvel exemple, passé de $Z$ dans les différents états du texte imprimé et que Pontieri, qui pourtant prend pour point de départ la leçon de B et dispose de C comme exemplaire de comparaison, n'a pas soupçonné: [...] ne spes aliqua recuperandi palatii uel, deducta uxore (C B : -tae -ris Z, edd.), procreandae propaginis reseruaretur (III 13 [p. 64, 28 sq.]). Nous retrouvons un cas analogue en I 10 (p. 13, 22 sq.): ruptisque muris aditis patentibus irrumpentes. Le solécisme aditis - que les éditeurs, singulièrement, ont tous admis sans douter, bien que cette forme de datif-ablatif ne soit pas autrement attestée - a été suggéré par les finales -is des deux ablatifs antécédents. Lire aditibus patentibus ( «se ruant par les brèches béantes qu'ils s'étaient ouvertes dans les remparts»). C'est le seul endroit de la chronique où aditus est au pluriel (il y a autant d' «accès» que de pans de murailles écroulés); en dehors de ce passage, le mot n'est employé qu'au singulier - le plus souvent dans une phrase négative. Cf. II 35 (p. 46, 5); II 40 (p. 49, 18); III 4 (p. 58, 25 sq.) ; III 12 (p. 64, 17); III 15 (p. 66, 7 sq.) ; III 20 (p. 69, 16 sq.) ; III 33 (p. 77, 24); IV 16 (p. 95, 36); IV 22 (p. 100, 40-101, 1); IV 24 (p. 102, 9 sq.); IV 25 (p. 104, 2); IV 26 (p. 105, 30 et 32). 
Sur nauibus, datif de lieu avec un verbe composé, cf. II 4 (p. 31, 9: praedam quam acceperat Regium deferendam nauibus < introduxit $>^{13}$ ); II 43 (p. 51, 37 sq. : quo facilior descensus ad mare equis nauibus introducendis fieret); III 8 (p. 61, 9: praedam nauibus inducunt $^{14}$ ); III 11 (p. 63, 28 sq. : [praedam] nauibus introducendam illorsum minare accelerat) et III 24 (p. 71, 16: copiis nauibus introductis).

II 5 (p. 31, 13 sq.) [...] Serlonem, nepotem suum, uidelicet Serlonis fratris sui filium cuius superius in fine primi libri mentionem fecimus, cum septem equitibus hostes appropriare $^{15}[\ldots]$ iubet, $[\ldots]$

$\|$ uidelicet - sui post filium transp. $B \|$ uidelicet $Z B$ : ut $C \|$ filium om. $C \|$ cum - iubet om. $Z B$, edd. $\|$

Si le choix de B comme fondement de la recension du texte de Malaterra explique pourquoi ce passage est tronqué dans l'édition d'E. Pontieri, il est étrange que le savant italien n'ait pas fait état dans son apparat du supplément dont $\mathrm{C}$ enrichit la tradition - manuscrite et imprimée ${ }^{16}$ - en restaurant la cohérence de cette fin du chapitre II $5^{17}$. Mais C a, lui aussi, subi le hasard des corruptions, et il n'est pas sûr en définitive que la lecture à adopter s'obtienne, comme M.-A. Avenel le laisse croire en donnant à imprimer le texte reproduit ci-dessus, en incorporant à la leçon de $\mathrm{Z}$ l'apport nouveau de C. Il est hors de doute que le ut de C représente uidelicet, qu'un copiste distrait n'aura pas reconnu sous une de ses formes abrégées. Mais filium, qui manque dans $C$ et que $B$ transporte devant uidelicet, a dû être sauté dans $\beta$ comme

13. Addition de M.-A. Avenel. Le verbe manquait dans l'hyparchétype commun (cf. C et Z). B a cru restaurer le texte en suppléant ponunt, que Pontieri a arrangé en posuit. Aucun de ces deux suppléments ne rétablit la correction grammaticale. Sur introducere + datif équivalant à in + acc., cf. Krebs \& Schmalz 1905, I, 778 .

14. Inducunt est un hapax dans Malaterra: il est permis de soupçonner que le chroniqueur, ici, avait écrit aussi introducunt - malgré VERG., georg. 1, 136 sq. : cum flauis messorem induceret aruis / agricola (autres exemples dans TLL VII 1, 1232, 9-16 [J.B. Hofmann, 1943]) ? «(introduco) variat in codicibus cum induco saepe » (TLL VII 2 fasc. 1, 66, 20-22 [Hugenschmidt, 1956]).

15. Lire appropiare, comme en I 16 (p. 16, 35: appropiare AC: appropinquare B approperare Z, edd.) et en IV 28 (p. 106, 28). Une confusion de même nature se répète dans Pontieri à propos des adverbes prope et propius, quand bien même la forme correcte en aurait été restituée dès l'édition princeps: cf. II 17 (p. 34, 20 : proprius); III 8 (p. 61, 10 : proprius); III 12 (p. 64, 16: proprius); III 37 (p. 79, 18 : propre $[$ sic] ); IV 16 (p. 95, 20 : proprius) et IV 17 (p. 96, 22 : proprius).

16. Ni Caruso, dans son édition de 1723 , ni Me ne signalent ici la lacune d'un des rameaux de la tradition.

17. Ainsi la lecture de $\mathrm{C}$ rend-elle inutile la correction de l'édition princeps, la substitution de praemissum à praemissis (p. 31, 15), raccord grammatical maladroit, mais indispensable pour donner à l'accusatif Serlonem qui, dans Z et B, reste en l'air, ne dépendant de rien, une apparence de consruction: Serlonem, nepotem suum, [...], ne, si fugere, sicut et fecerunt, uellent, liberius possent, sic praemissum ipse uelocius subsecutus, [...]. La subordonnée conjonctive énonçant l'objectif de la manœuvre est rapportée à praemissum, lequel forme avec Serlonem le complément d'objet de subsecutus. 
dans $\alpha$, puis rétabli conjecturalement dans $\beta$ et inséré à deux places différentes ${ }^{18}$. Devant cette variante d'ordre, M.-A. Avenel a donné la préférence à $Z$, le représentant le plus qualifié de la classe $\beta$. Il reste que ce filium, à l'endroit où $Z$ l'a introduit, a tout l'air d'un intrus: il rompt l'enchaînement grammatical naturel d'un nom et de son apposition. Comparer II 4 (p. 30, 22 sq.) [comes] obuium habuit quendam Sarracenum, militia inter suos nominatissimum, fratrem scilicet Bennecleri, pro cuius occisione Betumen a Sicilia eiectus fuerat; ou III 41 (p. 82, 15 sq.) eodem anno uenerabilis papa Gregorius, cuius superius mentio facta est, [...] obiit ${ }^{19}$. L'effet d'entrelacs créé par cette intrusion n'est pas attribuable à Malaterra ${ }^{20}$. Le texte originel est donc celui de B, qui présente un assemblage de mots, suum filium, dans lequel le substantif a pu être omis à cause du retour de - $u m^{21}$. Indice concordant: uidelicet n'introduit que rarement une incise explicative, Malaterra aimant à lui attribuer la seconde place $^{22}$ : on compte seulement cinq exemples de l'ordre contraire sur quelque trentequatre occurrences de l'adverbe ${ }^{23}$. Dernier indice - dont la valeur démonstrative est

18. Sur ces «fourvoiement [s] à place mobile» impliquant omission, voir Havet 1911, 356 .

19. Nous aurions pu mettre également en regard de notre phrase le morceau de texte suivant: (II 46 [p. 53, 27 sq.]) Serlo, filius Serlonis, nepos uidelicet utrorumque principum istorum, cuius etiam (om. $\mathrm{ZB}$, edd.) in praecedentibus mentionem fecimus, apud Ceramum morabatur, qu'on ne peut pas nous opposer. L'éloignement de la subordonnée n'est dû qu'à l'intercalation de deux appositions nominales qualifiant, comme la proposition relative - elle-même appositive -, le nom qui est l'antécédent du pronom relatif. Tout se suit de façon normale.

20. L'insertion de filium à la fin du syntagme ouvert par uidelicet répond à un usage bien établi chez les copistes d'installer en place finale les termes omis, puis restitués en marge par un correcteur: cf. Havet 1911, 361 .

21. Après fratris sui, l'omission de filium est sans vraisemblance. Le rapprochement des mss C et Z permet de conclure à une altération commune à toute la tradition. Mais la consécution suum filium prêtant à omission par saut du même au même, des copistes intermédiaires ont pu, chacun de leur côté, altérer le texte de leur modèle : la forme originale du texte, demeurée intacte dans B, a disparu dans $C$; un antigraphe de $Z$ a suppléé le terme sauté, mais celui-ci a été fourvoyé lors de l'exécution de l'exemplaire issu de la correction.

22. On peut en dire autant de scilicet: outre II 4 (p. 30, 23), cité ci-dessus, on relève II 1 (p. 29, 16 : animae scilicet et corporis) ; II 40 (p. 49, 19: mulierum scilicet et puerorum); II 43 (p. 51, 7 sq. : faces scilicet accendant). Font exception I 8 (p. 12, 6: scilicet ut; comparer IV 8 [p. 90, 20 : uidelicet ut]) et III 27 (p. 73, 33: scilicet quasi stadiis quatuor interpositis [" c'est-à-dire à une distance d'environ quatre stades »]). La même place seconde est réservée à quippe dans tous ses emplois comme particule explicative: cf. I 3 (p. 8, 13 et 8, 20 sq.) ; I 17 (p. 17, 17); I 40 (p. 25, 11) ; II 6 (p. 31, 24 sq.) ; II 19 (p. 35, 12 sq.) ; II 23 (p. 36, 35 [app.]); II 25 (p. 38, 11) ; II 36 (p. 46, 34 [app.]) ; II 39 (p. 48, 12); II 40 (p. 49, 10); III 3 (p. 58, 13); III 15 (p. 66, 14 sq.); III 29 (p. 75, 13 sq.); III 37 (p. 79, 21 sq.); IV 25 (p. 104, 8 sq.). Sur la disposition de ces trois mots adverbiaux chez les auteurs latins de l'Antiquité, voir Janson $1979,96-98 ; 115$.

23. Cf. III 27 (p. 73, 36 sq. : secundas uero [sc. uigilias], uidelicet a media nocte donec diescat); III 31 (p. 76, 14 sq. : uxorem nepotis sui Serlonis, uidelicet qui apud Siciliam a Sarracenis interemptus fuerat); IV 8 (p. 90, 20 : uidelicet $u t$ ); IV 20 (p. 99, 11: salua fidelitate legalium heredum suorum, uidelicet fratris) et IV 26 (p. 104, 24 sq. : Adala, uidelicet marchionis Flandrensis filia). Mais III 31, IV 20 et IV 26 
sans doute moins contraignante, le latin de Malaterra étant une langue artificielle: le complément déterminatif au génitif indiquant la filiation est en règle générale postposé. Le chroniqueur a usé vingt-huit fois de la formule «un(e) tel(le), fils / fille d'un tel » : dans cinq cas seulement - dont il est difficile d'apprécier la valeur stylistique -, le génitif déterminatif se place devant le mot qu'il détermine ${ }^{24}$. On observe donc en II 5 la même ordonnance des termes qu'en III 28 (p. 74, 29 sq. : neptem [...] habens, filiam uidelicet fratris sui Guillelmi, Principatus comitis), IV 14 (p. 93, 16 : neptem Bonifacii [...], filiam uidelicet fratris eius) et IV 21 (p. 99, 21 sq. : sororem ducis, filiam uidelicet Guiscardi) : Serlonem, nepotem suum, filium uidelicet fratris sui, cuius superius [...] mentionem fecimus, [...] hostes appropiare [...] iubet.

\section{9 (p. 32, 9) diuinum inuocat auxilium.}

$\|$ inuocat $B$, Pontieri Avenel: inuitat $C Z \|$

Sans doute peut-on s'autoriser de certains précédents ${ }^{25}$ pour maintenir ici, aux dépens de la lecture traditionnelle, cette innovation de Pontieri. Mais en bonne méthode, l'accord des deux sources indépendantes $\mathrm{C}$ et $\mathrm{Z}$ commande de récuser le témoignage de B. D'autre part, si inuocat est bien le terme convenu en pareil contexte - les chefs normands invoquent l'assistance divine à l'heure du danger ${ }^{26}-$, on ne voit pas quelle circonstance particulière aurait déterminé deux copistes isolés à

semblent devoir être éliminés; car on peut redonner à uidelicet sa position normale, soit en réformant la ponctuation de l'édition Pontieri : uxorem nepotis sui, Serlonis uidelicet, qui apud Siciliam a Sarracenis interemptus fuerat (III 31); non uiliori legato quam laterali coniuge, Adala uidelicet, marchionis Flandrensis filia (IV 26), soit en substituant au texte traditionnel la leçon héritée du subarchétype: salua fidelitate legalium heredum, filiorum (C B : suorum Z, edd.) uidelicet (C Z: scilicet B) fratris (IV 20).

24. Cf. III 10 (p. 62, 3 sq. : Iordanum, comitis filium); IV 23 (p. 101, 15 sq. : Corradum, eiusdem Henrici filium); IV 26 (p. 104, 17: Ricardus iuuenis, Iordani principis filius); IV 26 (p. 104, 24 sq. : Adala, uidelicet marchionis Flandrensis filia) et IV 26 (p. 104, 27 : sororis uidelicet filius). À l'époque historique, l'antéposition du génitif est habituelle dans la formule de filiation : cf. Kühner et al. 1976, II 2, $610, \$ 246.9$.

25. Cf. Hirt. ap. Caes., Gall. 8, 48, 3; Liv. 22, 14, 8; VAl. Max. 1, 8, 12; Sen., epist. 52, 7 ; TaC., ann. 1, 75,$2 ; 15,59,3$; CoD. Iust. 1, 17, 1, 2. La Bible ne fournit aucun parallèle textuel véritable, l'idée du secours qu'on implore étant subordonnée à la personne de Dieu : cf., par exemple, $J g$ 6, 7 clamauit ad Dominum postulans auxilium contra Madianitas ; 1 Ch 5, 20 quia Deum inuocauerunt cum proeliarentur; Si 46, 6 Inuocauit Altissimum potentem in obpugnando inimicos undique; ou $2 M_{13}$, 10 quibus Iudas cognitis praecepit populo ut die ac nocte Dominum inuocarent quo sicut semper et nunc adiuuaret eos. Il en va de même quand on sollicite l'assistance d'un puissant de ce monde: cf. 2 Ch 28, 16 misit rex Achaz ad regem Assyriorum auxilium postulans.

26. Cf. II 33 (p. 43, 32: Deum sibi adiutorem inuocans) et III 30 (p. 76, 4: certamen, Deum sibi propitium inuocantes, ineunt). Les trois autres emplois d'inuocare sont repris de l'apôtre Paul, dont une formule $(R m 10,12)$ est citée et exploitée dans un excursus théologique en II 33 (p. 43, 14 sq. et 43, 16 sq. [bis]). 
altérer la leçon de leur modèle respectif pour insérer l'un et l'autre dans la tradition le même terme réputé impropre ${ }^{27}$. La rencontre fortuite des copistes de $\mathrm{C}$ et $\mathrm{Z}$ sur inuitat est tout à fait invraisemblable, cette leçon fût-elle mieux représentée dans Malaterra que sa variante; c'est B plutôt qui est suspect de révision. Sans doute ne peut-on pas défendre la lecture de $\mathrm{C}$ et de $\mathrm{Z}$ par des exemples tirés de la chronique elle-même: dans tous ses autres emplois, inuitare est construit invariablement avec l'accusatif de la personne dont on requiert l'intervention; cf. II 25 (p. 38, 14 sq.) inuitant fratrem ut fratri $[. .$.$] auxilium ferendo subueniat { }^{28}$; IV 10 (p. 91, 11) auunculumque sibi in auxilium inuitans; IV 17 (p. 96, 11 sq.) auunculum comitem a Sicilia ut sibi auxilium ferendo illuc occurrere non differat inuitat; IV 26 (p. 104, 23-25; l'accusatif comitem doit être suppléé) Dux [...] ut cum exercitu uenire non differat ac sibimet auxilium laturus summopere inuitat ${ }^{29}$. Ce n'est pas toutefois une raison suffisante pour condamner le tour. D'autres auteurs fournissent des exemples comparables, prouvant indubitablement qu'inuitare, comme inuocare ${ }^{30}$ ou implorare ${ }^{31}$, admet les deux types de complément direct, animés et abstraits: cf. par exemple Avg., ciu. 3, 6 (p. 102, 31-103, 1 Dombart-Kalb [à propos de Romulus]) : [...] deorum tutelam Romanis inuitauit iste sui fratris extinctor? ou Oros., hist. 7, 37, 10 : quia [sc. Radagaisus] fauorem deorum sacrificiorum obsequiis inuitaret ${ }^{32}$.

Il n'y a pas lieu donc de prendre le parti de l'innovation et de donner à inuocat la préférence sur inuitat. inuocat n'est qu'une leçon particulière de B; et l'accord de $\mathrm{C}$ avec Z, le représentant le plus autorisé de la classe adverse, suffit bien ici pour déterminer de quel côté est le texte authentique.

27. Cette disconvenance avec le contexte n'est point apparue à Caruso, qui omet de relever la variante de B.

28. Cf. Index II xxv (p. 28, 2) A militibus ducis comes ut fratri succurrat inuitatur.

29. La construction de ce membre de phrase ne fait difficulté qu'en apparence: la coordination $a c-B$ donne $a d$, inutilisable; A et $\mathrm{C}$, mutilés et incomplets, sont défaillants - fait se répondre une subordonnée complétive finale, ut [...] non differat, et un participe futur, laturus. Comparer IV 21 (p. 99, 30 sq.) nec [...] congauisurus nec ut urbem [...] reddat accedere curauit.

30. A en juger par l'usage du seul Malaterra, l'inuocat auxilium de B n'a pas en soi plus d'autorité que l'inuitat auxilium de C Z: la chronique n'offre aucun morceau de texte parallèle où l'un de ces deux verbes prenne pour complément direct la chose qu'on sollicite.

31. Cf. II 9 (p. 32, 15) Deum ordinatorem et fortiorem gubernatorem [...] implorant; IV 25 (p. 103, 33) Dei auxilium implorans. Notons en passant qu'en Index II IX (p. 27, 11 : Nostri, transitum sibi turbari uidentes, adiutorem implorant), M.-A. Avenel a raison de rétablir Deum auprès d'adiutorem : il n'y a pas d'exemple que Dieu soit l'Adiutor par excellence, comme Paul est l'Apostolus ou Averroes le Commentator; comparer, outre II 9 (p. 32, 12 [citation non identifiée]) et II 33 (p. 43, 32), Ps 29, 11 Dominus factus est adiutor meus; 51, 9 Ecce homo qui non posuit Deum adiutorem suum ; 2 M 12, 36 Inuocauit Iudas Dominum adiutorem; etc.

32. Comparer Аrnoв., nat. 1, 49, 1: cum Aesculapium ipsum [...] inuitarent miserrimis uotis. 
II 10 (p. 32, 17 sq.) [...] ac si legisset: «Quid refert? Armis contingat palma dolisue», eqs.

$\|$ post legisset add. illud $C Z B \|$ quid $e d$. pr. Pontieri : nil $C B$ nil aut quid dubit. $Z^{2}$ ius $Z^{1} \|$ contingat $C Z$ : confirmat $B \|$

Il faut rétablir illud, que Pontieri passe sous silence, et substituer nil à quid, suggéré par Zurita $\left(=Z^{2}\right)$ pour remédier à l'incompréhensible ius de sa source. Nil et quid sont indifférents au point de vue de la métrique; mais quid n'a que la valeur relative d'une conjecture: seul, nil est authentique. Illud introduit une citation textuelle de Prudence, psych. $550^{33}$; commencer cette citation avec le démonstratif revient à restreindre son rôle cataphorique ${ }^{34}$, et on dénature l'hexamètre originel en l'augmentant d'une mesure.

II 12 (p. 33, 7 sq.) Sicque mari hostibus purgato, patenti absque periculo transitu, dux cum omni exercitu placido cursu transmeans Messanam uenit.

\| patenti om. $B \|$ periculo $Z B$, edd. : -loso $A C \| \operatorname{cum} C Z B$ : enim $A \|$ placito $C$ $Z$ : placido $A$ B, Pontieri Avenel $\|$

Tel est le texte que l'édition princeps a établi d'après le ms. $Z$ et que tous les éditeurs sans exception se sont transmis, même quand ils disposaient du contrôle d'un des représentants de la meilleure des deux recensions (A ou C). Ainsi Pontieri n'indique que l'omission de patenti dans B et dans un de ses descendants, D, ses témoins de prédilection. Ni Caruso ni Me ni Pontieri ne relèvent les variantes periculoso et placido / placito, comme si le texte de la vulgate était inattaquable et qu'il leur apparût que les leçons divergentes n'étaient que d'insignifiantes erreurs de copie. Si M.-A. Avenel les a dûment enregistrées, elle adopte sans défiance la lecture traditionnelle. Et pourtant il est permis de s'interroger sur l'authenticité du periculo de

33. La citation a été identifiée par Wolf 2005, 90. Du coup, le traducteur américain s'est affranchi de la ponctuation aberrante de l'édition Pontieri, laquelle, au rebours de ses devancières, isole par un point d'interrogation le verbe principal de son régime et scinde la phrase en deux propositions indépendantes. Le sens littéral de ce vers ne fait pas de doute; s'il a été parfois mal rendu, c'est à cause de la présence de ce signe - outre que le poète recourt ici à une forme simplifiée de l'interrogation indirecte dite double: le -ue de dolisue (cf. Hofmann-Szantyr 1965, 545, \$296 II). Ainsi G. A. Loud escamote-t-il purement et simplement le subjonctif contingat: "What is to be done? Success falls to the crafty weapons"; de son côté, Lo Curto 2002,101, prête à ce subjonctif la valeur que revêt ce mode dans les subordonnées introduites par dum ou modo: "Che importa? Con le armi o con l'inganno, purché si raggiunga la vittoria». Le texte de Pontieri - ponctué selon la grammaire et le sens - pouvait se rendre comme suit: "Qu'importe si la victoire est obtenue par les armes ou par des ruses?».

34. En transportant le pronom après les deux points, M.-A. Avenel en borne le champ d'application à la seule subordonnée interrogative indirecte (cf. Avenel 2001, II, 72). - C’est un emploi isolé dans la chronique ; ailleurs, le démonstratif s'associe avec d'autres formules : cf. par exemple p. 4, 31 (épître dédicatoire) His competenter assignari potest illud Scripturae, qua dicitur; I 26 (p. 20, 31) quasi naturaliter sciens illud Sallustianum prouerbium (pour introduire une citation légèrement déformée et augmentée de SALL., Iug. 3, 3); ou II 7 (p. 31, 28) propter illud quod dicitur. 
$\mathrm{ZB}$, dont le periculoso de AC ne peut être a priori provenu ${ }^{35}$. Sans doute n'a-t-on pas prêté assez d'attention au détail : rapprochés l'un de l'autre par la construction, patenti et absque periculo sont passablement redondants ${ }^{36}$. Cette répétition d'idées n'existe pas dans la construction concurrente. C'est le danger constitué par la présence de la flotte panormitaine qui empêchait le débarquement des Normands sur la côte sicilienne. Les Arabes ayant vidé les lieux, la voie est libre : la mer est dégagée - mari $[\ldots]$ patent $i^{37}$-, et la traversée est sans danger - absque periculoso transitu. Ce qui donne en traduction: "Ainsi, la mer, une fois purgée des ennemis, se trouvant dégagée, le duc arriva à Messine, avec l'ensemble de ses troupes, sans avoir été mis en danger durant la traversée [...] ». Il est moins facile de choisir entre les variantes placido et placito $^{38}$. placido a passé de B dans la tradition imprimée sur l'initiative de Pontieri; mais le participe ne donne pas un sens moins satisfaisant que l'adjectif: «([litt $\left.{ }^{\mathrm{t}}\right]$ étant passé <en Sicile> au terme d’une navigation) agréable ».

\section{19 (p. 35, 12 sq.) speciosa quippe et praeclari generis erat $\|$ generis $Z B$ : germinis $A C \|$}

Si l'on se réfère à la classification des manuscrits, la leçon autorisée est le germinis de AC, qui donne un sens excellent ${ }^{39}$, non le generis que ZB lui a substitué ${ }^{40}$ et

35. Nous ne croyons pas au pur lapsus : la transformation du substantif en adjectif modifie la distribution des termes de ce membre de phrase; désormais patenti se rapporte à mari et absque régit transitu. Ce n'est pas la manière de procéder de $\mathrm{AC}$, respectueux - parfois jusqu'à l'absurde - du texte transmis. En revanche la substitution inverse est un de ces arrangements volontaires qui ont permis de conclure à la révision de $\beta$ par un correcteur. La révision a porté non seulement sur des fautes - soit propres soit communes à toute la tradition -, mais aussi sur des morceaux de texte mal compris. Ici, mari étant déjà pourvu d'une détermination, patenti a été mécaniquement attiré par transitu, et periculoso a, par contrecoup, été retouché pour fournir à la préposition absque son régime. Le procédé ne manque pas d'habileté et il témoigne d'un certain souci de l'équilibre formel : il produit deux syntagmes de même étendue, dont les éléments constitutifs s'opposent en chiasme. Au chiasme qui se remarque dans $\mathrm{Z}$ - la figure n'existe plus dans B, du fait de la chute de patenti -, AC substitue une enclave; comparer II 40 (p. 49, 18) nullo ad introducendum [sc. uictum], hostibus circumsertis, aditu patente; III 4 (p. 58, 25 sq.) nullo aditu ad uictum introducendum, se suisque negantibus, patente; III 41, (p. 82, 22) Graecia hostibus recedentibus libera laeta quieuit.

36. Dira-t-on que Malaterra n'était pas à une redondance près? Après ce début de phrase - de quelque manière qu'on le construise -, la nécessité du groupe participial placido / placito cursu transmeans ne se révèle pas immédiatement à l'esprit...

37. Cette forme isolée a l'air d'une faute, qu'il faut sans doute corriger, d'après l'analogie des autres passages, en patente: cf. II 35 (p. 46, 5); II 40 (p. 49, 18); III 4 (p. 58, 26); IV 26 (p. 105, 30).

38. Elles se trouvaient vraisemblablement dans le plus proche commun ancêtre de tous nos manuscrits, lequel devait porter l'une de ces formes dans le texte, et l'autre comme variante interlinéaire ou surcharge corrective. C'est pourquoi l'une et l'autre leçon se retrouve dans les deux rameaux de la tradition.

39. Cf. TLL VI fasc. 2 IX, 1924, 22 : «i. q. origo, genus, gens» (Schuster, 1930), et Lavarenne 1933, 449.

40. La substitution inverse est bien moins probable: pourquoi generis se serait-il altéré, puis transformé en germinis, un terme moins banal et d'un usage moins courant en pareil contexte? Sauf erreur 
qui semble en être une glose explicative ${ }^{41}$. Malaterra a pu emprunter le mot à des livres, anciens - comme Prudence ${ }^{42}$, saint Jérôme ${ }^{43}$ ou Boèce ${ }^{44}-$ ou modernes comme Dudon de Saint-Quentin ${ }^{45}$ ou Guillaume de Poitiers ${ }^{46}$.

II 23 (p. 36, 29 sq.) ubi, insolito aere corrupto, ipse nihilominus infirmatus, quosdam suorum amiserat.

$\|$ corrupto $Z B$ : -tos $C \|$

Si insolito aere et aere corrupto sont intelligibles ${ }^{47}$, l'enchaînement des trois termes cesse de l'être ${ }^{48}$. Même si les traductions ne permettent guère d'en voir la difficulté, l'association de l'adjectif et du participe ne laisse pas d'être insolite et, partant, suspecte. Au contraire le corruptos de C, qui ne figure pas dans l'apparat de Pontieri,

de notre part, le mot ne se rencontre pas ailleurs dans la chronique. Néanmoins, sa rareté ne suffit pas à condamner ce doublet: on ne peut pas exclure un mot du stock lexical d'un auteur sous prétexte que c'est un hapax. Sur genus désignant la naissance, cf. I 4 (p. 9, 7 et 9, 9) ; I 30 (p. 22, 14); III 10 (p. 61, 29); III 28 (p. 74, 24); III 31 (p. 76, 13; 76, 17; 76, 20 et 76, 21); IV 8 (p. 90, 7).

41. Une fois de plus Pontieri conserve telle quelle la leçon traditionnelle et néglige de reporter dans l'apparat la variante concurrente. Il faut croire que C aura été, au rebours de B, fort mal collationné. Sur l'exactitude toute relative des collations de Pontieri, cf. Resta 1964, 16 sq. (P [=ed. pr.]); 22 sq. (D) ; 24 (A) ; 27-29 (B); 29-32 (C).

42. Cf. apoth. 418: [Iesu] nate Deo, nate sceptris et germine Dauid; perist. 3, 1 sq.: Germine nobilis Eulalia / mortis et indole nobilior [...] ; etc. « Mot favori de Prudence: on en relève dix-huit exemples» (Lavarenne 1933, 449).

43. Cf. epist. 66, 6: consulum pronepos et Furiani germinis decus.

44. Cf. cons. 3, 6 carm. 6 : mortales [...] cunctos edit nobile germen.

45. Cf 102 (p. 265, 2 Lair, v. 16 sq.) : quaque [sc. uirgine] uergente tempore dignus / nascetur haeres germinis almi. Autres occurrences du mot dans Dudon: 102 (p. 264, 10 Lair) et 121 (p. 285, 9 Lair). Nous devons ces références à Pierre Bouet, notre mentor devenu notre ami, que nous remercions d'avoir bien voulu interroger pour nous la concordance de Dudon qu'il s'est confectionnée.

46. Cf. Histoire de Guillaume le Conquérant, I 22 (p. 46 Foreville) : [...] Baldwinus nobilitate item prisco ex germine tradita illustrissimus. Le récent Lexique Latin-Français, Antiquité et Moyen Âge de M. Parisse et M. Goullet (Paris, Picard, 2006) ne mentionne pas cette acception du mot.

47. Columelle, Sénèque ou Tacite, par exemple, incriminent eux aussi l'" air vicié» dans le déclenchement des maladies: cf. Colvm. 1, 4, 9 circumfusus aer corruptus plurimas adfert corporibus nostris causas offensarum; SEN., nat. 6, 27, 2 aer [...] corruptus internorum ignium uitio [...] purum hunc liquidumque maculat ac polluit; TAC., hist. 5, 7, 2 halitu lacus [...] corrumpi superfusum spiritum [...] reor. Sénèque encore, à propos des miasmes qui, libérés par de violents tremblements de terre, provoquent des épidémies, use d'une formulation proche de l'insolito aere de Malaterra: cf. nat. 6, 27,2 aer $[\ldots]$ corruptus $[\ldots]$ insuetum ducentibus spiritum affert noua genera morborum. Même attention prêtée par le chroniqueur aux effets du climat méditerranéen sur les Normands en I 16 (p. 16, 8 sq.).

48. Dira-t-on que ceux des Normands qui ont succombé lors de ce déplacement du comte Roger à Gerace «ne s'habituaient pas à l'air vicié »? Aucune des traductions que nous avons pu consulter n'a pris ainsi le latin des éditions à la lettre. Cf. Loud, ad loc. : «through some unknown contagion in the air»; Lo Curto 2002, 117 : "a causa di un insolito inquinamento atmosferico»; Wolf 2005, 97 : "due to the unusually bad air in that region». 
ne présente aucune ambiguïté de construction et permet de rétablir le sens : certains hommes de Roger ont été contaminés - corrupt $[i]^{49}$ - par l'atmosphère du lieu, probablement malsaine, mais que Malaterra caractérise en appuyant sur l'idée que les Normands ne parviennent pas à s'y habituer (insolito aere).

Le corrupto de ZB s'explique aisément par la suggestion du double ablatif qui précède et le relatif éloignement du pronom auquel il doit être rapporté (quosdam).

II 26 (p. 38, 21 sq.) [...] et quia fratrem meum mihi inimicari et Geracium me obsessum uenientem cognouistis, captum in mea fidelitate tenetis, gratissimum habeo.

$\|$ et - mihi om. ZB, ed. pr. || post et add. quod Avenel || mihi - me om. Pontieri $\|$ inimicari $C Z$ : -cantem $B \|$ geracium $M e$ : gergentium $C$ germanum $Z$ gerentem $B$ post corr. $B$ ante corr. non legitur $\|$ me $C Z M e$ : meum $B \|$ post captum add. et Pontieri $\|$

Sous quelque forme qu'on l'examine - la version tronquée qu'en propose l'édition Pontieri ${ }^{50}$ n'en estompe pas la singularité -, ce morceau de texte présente une construction en apparence brouillée. Le sens causal de la subordonnée quia-cognouistis s'impose a priori; et cette cause se rapporte naturellement à captum - tenetis, non à gratissimum habeo. On ne voit pas, en effet, quelle gratitude on peut mériter à être au courant de quelque chose. En revanche, il est légitime que le comte Roger sache un gré infini - gratissimum habe $[t]$ - aux habitants de Gerace d'avoir neutralisé et réduit à sa discrétion un ennemi déclaré. L'insertion d'un quod devant quia, comme le préconise M.-A. Avenel ${ }^{51}$, rétablit donc une forme de cohérence logique et syntaxique. Mais on peut aussi penser que cette fin de phrase, telle qu'elle a été transmise et longtemps acceptée ${ }^{52}$, est exacte : non seulement quia introduit la

49. Sur corrumpere pour désigner une dégradation physique par la maladie, cf. par exemple Lvcr. 3 , 503 ; Cels. 2, 10 ; Tert., resurr. 57 (p. 117, 8-10 Kroymann); et le Mittellateinisches Wörterbuch, II, p. 1940, 13-19, s. v., II 2.

50. L'éditeur italien n'a pas su tirer parti de toutes les données nouvelles de C. Sa méthode est proprement incompréhensible : il retient - légitimement - ce qui, dans $\beta$, a été perdu, selon toute apparence, par saut du même au même, mais il exclut les premiers mots sur lesquels les deux états de la tradition se rejoignent après la lacune (inimicari et girgentium / germanum). D'autre part, on ne voit pas comment, après l'addition d'un et derrière captum, il construisait et interprétait la double subordonnée quia fratrem meum obsessum uenientem cognouistis captum et in mea fidelitate tenetis. Enfin il manque à ses obligations d'éditeur : en ne consignant dans l'apparat que la lecture de l'édition princeps $(\mathrm{P})$, il donne à entendre que le texte qu'il imprime est celui de ses sources manuscrites $(\mathrm{B}, \mathrm{D}$ et $\mathrm{C})$ et que celles-ci sont unanimes.

51. Cf. "La nouvelle édition de la chronique de Geoffroi Malaterra», in Primo rapporto sull'edizione delle fonti del Mezzogiorno medievale (Atti del convegno internazionale, Napoli 11-12 aprile 2003), n. 19. Nous remercions M.-A. Avenel d'avoir bien voulu nous adresser, avant impression, la version définitive de sa contribution à ce colloque.

52. Mais peut-on faire crédit aux uett. edd. pour la compréhension parfaite de cette phrase? Les versions modernes, confrontées au texte difficultueux de Pontieri, subordonnent à gratissimum habeo 
subordonnée causale dont le verbe est cognouistis, mais il doit être suppléé - sans qu'il soit besoin de donner un sens nouveau à la conjonction ${ }^{53}$ - en tête de la proposition dont le verbe est tenetis pour former le complément de gratissimum habeo. Sur cette ellipse, voir Stotz 1998, 391 sq., \$102.

II $30(\mathrm{p} .41,2)$ [comes] pluribus interemptis, sola dextra et Dei adiutorio liberatur $[\ldots]$.

|| interemptis edd: interceptis Z -ceptus C B \|

interemptis, qui se présente sans variante dans l'édition Pontieri, comme si tous les manuscrits s'accordaient en faveur de cette lecture, fournit sans doute un sens excellent, mais la correction ne s'impose pas. Elle serait même arbitraire, si l'on s'en rapporte au scholiaste de Suétone et à divers commentateurs, qui nous rappellent que, dans certains de ses emplois, intercipere correspond à occidere. L'inutilité de la conjecture est démontrée par des exemples comme Svet., Iul. 20, 5 (sur l'exécution de Vettius) : intercepisse ueneno indicem creditur; Plin., epist. 6, 25, 4 (sur la disparition de Metilius Crispus et de ses gens) : Interceptusne sit a suis an cum suis dubium; TAc., Agr. 5, 2 (p. 4, 2 sq. Delz): trucidati ueterani, incensae coloniae, intercepti exercitus $^{54}$; Tert., apol. 25, 5: pro salute Marci iam intercepti (répondant à Marco Aurelio apud Sirmium subito interempto); Avr. Vict., Caes. 3, 3: [...] quos [sc. la mère et les frères de Caligula] uario Tiberius exitio interceperat; etc. On peut même citer à l'appui de ce passage une phrase du chapitre II 5 où ce verbe est généralement - mais à tort - entendu dans un sens différent : ita [Messanenses] intercepit ut uix ex tanta multitudine unus euaserit (p. 31, 15 sq. $)^{55}$, à quoi s'ajoute III 30 (p. 75, 31-33), que

les deux propositions coordonnées quia-cognouistis, captum et - tenetis, dont elles font ses complétives, sans instituer entre elles d'autre hiérarchie qu'un rapport de succession chronologique.

53. Comparer II 40 (p. 49, 9) et quia a uobis uoluntarie mihi praesentantur, gratum habeo (= « et je vous sais gré de bien vouloir de votre propre initiative les exposer à ma vue»). C'est le seul autre exemple de cette construction dans la chronique; néanmoins il tend à prouver que, pour introduire la complétive qui exprime l'action que l'on «tient pour agréable» ou «digne de reconnaissance» (cf. Moussy 1966, 170-172), Malaterra recourait à quia de préférence à quod, privilégiant la nuance causale, toujours sensible dans ce tour. Hormis II 33 (p. 43, 10 sq. et p. 43, 17 sq. [quia C ZB : quod Pontieri]), deux endroits où la particule, jointe à l'indicatif, prend la place d'une proposition infinitive, quia est exclusivement une conjonction causale.

54. Ogilvie \& Richmond 1967, 147, qui défendent la leçon des mss, font l'observation suivante: «The emendation intercepti (as in c. 28, 3 and 43, 2), which would mean "destroyed", is unnecessary"; cf. Boetticher 1830, 283: " [...] latius sane patet huius verbi usus ap. arg. aet. scriptt., cum haud raro exprimat subito interficere, praematura morte abripere [...]».

55. En effet, pour parvenir au résultat constaté, il ne suffisait pas seulement d'arrêter l'ennemi dans sa fuite et lui couper la retraite. Nous comprenons plutôt: « [le comte Roger] tua tant d'ennemis qu'un seul à peine en réchappa sur un si grand nombre » - interprétation que confortent les premiers mots du chapitre suivant (p. 31, 17): Messanensibus suorum funera flentibus; cf. ibid. (p. 31, 19 sq.) : quamuis paucis qui adhuc supererant. III 26 (p. 72, 32 : ut [...] facilius intercepti perimerentur) 
M.-A. Avenel nous a signalé - et que Pontieri, cette fois, a enregistré dans son apparat: Elias Cartomensis, [...] ex Sarracenis ad fidem Christi conuersus, postea apud Castrum Iohannis a sua gente hostiliter interceptus (C Z, ed. pr. : interfectus B, Pontieri) [...].

II 32 (p. 41, 41-42, 1) comperto quod Arabici et Africani [...] apud Castrum Iohannis usque ad quingentos morarentur, [...]

$\|$ usque om. $B \|$ quingentos $Z B$ : - ti $C \|$

De même que uel certe, dans Malaterra, a très souvent dépouillé tout caractère restrictif et ne se distingue guère du simple uel disjonctif ${ }^{56}$, usque ad devant un nom de nombre n'a pas plus de valeur que n'en a $a d$ seul $^{57}$, et la locution est employée à peu près de la même manière que les adverbes $a d$ ou $\operatorname{circa}^{58}$ en latin classique. Il ne serait pas difficile de mettre en regard de chacune des dix phrases de Malaterra ${ }^{59}$ présentant la locution adverbiale un passage de César ou de Tite-Live où l'approximation devant un nom de nombre est exprimée par le truchement du seul $a d^{60}$.

Quant au quingentos de ZB, qui est la leçon traditionnelle, il semble n'avoir aucune autre autorité que celle de la chose imprimée. Et si Pontieri pouvait se laisser prendre par la quasi-unanimité de ses sources (B, D, P [= ed. pr.]), M.-A. Avenel aurait dû appliquer strictement les principes d'édition énoncés par G. Resta et imprimer quingenti, que donne $\mathrm{C}$. Il est improbable qu'après $a d$, une forme sentie comme un accusatif ait été changée, par une intervention de copiste, en un nominatif. En

est un autre cas : le sens de «tuer » étant réservé à un verbe dont c'est la valeur propre (perimerentur), intercepti veut bien dire « une fois leur retraite coupée».

56. Même emploi dégradé de certe pour introduire le second membre d'une interrogation indirecte double en I 3 (p. 9, 2 sq. : nescimus utrum in praecedentibus partibus uel certe in postea futuris heredibus aut etiam in utrisque [...]) et II 26 (p. 38, 38 sq. : dubii utrumne ea [...] ex industria [...] aut certe ex uero odio dicerentur [...]).

57. Malaterra a usé du simple ad une seule fois, en II 33 (p. 44, 32 : pedites ad uiginti milia [...] oppugnantes).

58. On ne compte dans la chronique que deux exemples de circa, employé à chaque fois comme préposition : cf. II 31 (p. 41, 31 sq.) coepit esse sollicita circa castrum tuendum (au lieu de de + abl. dans le sens de «en ce qui concerne, relativement à»), et II 40 (p. 49, 31-33) Ducis uero ministri [...] noctis excubias solito attentius uigilesque circa ducem deputant.

59. Cf., outre II 32 (p. 41, 41), I 7 (p. 11, 16); I 9 (p. 12, 18); I 16 (p. 16, 16) ; II 17 (p. 34, 18) ; II 33 (p. 44, 29) ; III 11 (p. 63, 30); III 12 (p. 63, 42); III 20 (p. 69, 10); III 30 (p. 76, 1).

60. Ainsi peut-on mettre en parallèle I 9 (p. 12, 18 sq. : maxima multitudine [...] coadunata, usque ad sexaginta milia armatorum) et LIv. 31, 21, 18 (Placentini captiui, ad duo milia liberorum capitum, redditi colonis); I 16 (p. 16, 16: usque ad sexaginta quos Sclauos appellant [...] secum habens) et Liv. 29, 34, 6 (iam ad quattuor milia equitum habebat); II 17 (p. 34, 18 : quos caedendo uersus Castrum Iohannis persequentes usque ad decem milia occiderunt) et Liv. 29, 34, 16 (ceteros [...] effuse fugientis [...] uictores secuti ad duo praeterea milia equitum aut ceperunt aut occiderunt); II 33 (p. 44, 29) et Liv. 27, 12, 16 ou 31, 28, 2 ; III 20 (p. 69, 10) et Liv 24, 7, 2 ou 26, 47, 2 ou encore $27,8,13$. De toute évidence, usque est joint par une sorte de pléonasme à l'adverbe $a d$, qu'il renforce sans rien conserver de sa valeur première. 
revanche, l'intervention inverse, qui régularisait en apparence la syntaxe, est tout à fait plausible - surtout venant du correcteur de $\mathrm{ZB}^{61}$.

II 33 (p. 43, 8-10) Si autem cum propheta ${ }^{62}$ admirantes requirimus "quomodo persequebatur unus mille» [...], profecto nobismetipsis ex eiusdem prophetae uerbis absque mendacio respondere possumus [...]

$\|$ si $C Z$ : quid $B \|$ cum propheta $C Z$ : prophetam $B$, Pontieri dicentem post requirimus addentes $\|$ nobismetipsis $Z B$ : nobiscumipsis $C \|$ ex $Z B$ : et $C \|$

nobismetipsis est une innovation inopportune de $\mathrm{ZB}$ qu'il convient d'écarter. On lui substituera la leçon que porte $\mathrm{C}$ - mais que Pontieri ignore purement et simplement. En effet ce nobiscumipsis n'est qu'une extension au verbe respondere du tour secum / cum animo usuel avec les verbes cogitandi, "quibus designatur tacita et occulta cogitatio, qua aliquis non cum aliis agat, sed se cohibens aut in se conclusus. Quare additur saepe ipse ${ }^{63}$ - tour représenté en I 17 (p. 17, 22) Quod secum diu reuoluens, II 1 (p. 29, 19) Haec secum animo reuoluens, et III 12 (p. 64, 8 sq.) secum tacitus agebat $^{64}$ quibus artibus [...] uicissitudinem recompensaret. Dans cette digression ouverte en plein récit à l'occasion d'un brillant exploit de Serlon le Jeune, que le narrateur met expressément en corrélation avec un verset de la Bible évoquant une situation en tout point comparable, nobiscumipsis est plein de sens : "Et si, saisis d'étonnement, nous nous demandons avec le prophète: "Comment donc un seul homme en met-il mille en fuite ?” [...], nous pouvons assurément, sans trahir la vérité, nous faire intérieurement la réponse suivante, en reprenant les mots de ce prophète $[\ldots]$ ».

61. Comparer Liv. 22, 50, 11 ad sescenti (-tos ed. Venet. 1518) euaserunt. Cf. Walters-Conway ad loc. et ad 22, 41, 2; Weissenborn-Müller ad 3, 15, 5 et 10, 33, 6 .

62. Ici, Pontieri a accueilli par distraction une leçon propre de B - reportée incorrectement et sans variante dans l'apparat -, prophetam [...] dicentem, sans s'apercevoir qu'elle était tout bonnement impossible. L'accusatif prophetam et l'interpolation de dicentem sont le résultat d'un arrangement grossier d'un état antérieur du texte de cette recension altéré par la chute de cum. Cette double correction donne un semblant de construction - requiro n'est pas rogo, et il ne prend jamais dans Malaterra l'accusatif de la personne à qui l'on demande (cf. II 21 [p. 35, 28-36, 2]; III 38, v. 11 [p. 8o, 35] ; IV 24 [p. 102, 12]) -, mais elle introduit un non-sens que la considération du contexte suffisait à dénoncer. Non seulement dicentem rompt le lien grammatical unissant le verbe requirimus à son régime direct, quomodo persequebatur unus mille, mais il est inutile et indéfendable. Aucun des traducteurs de Pontieri ne rend d'ailleurs le mot-à-mot, dénué de sens.

63. Hand 1832, 150. Cf. TLL IV Con-CyvLvs, 1376, 19 sq. (Hey, 1909) «cum verbis loquendi et cogitandi (secum fere i. q. «in animo suo»)».

64. aiebat $(\mathrm{C}$, Pontieri) est une faute évidente, imputable à un copiste, et il faut rétablir agebat ( $\mathrm{ZB}$, ed. pr. [= « il réfléchissait, sans rien en dire, aux moyens de lui rendre la pareille»]; comparer III 13 [p. 65, 10] tacitus agebat). Sur la confusion de ces deux graphèmes, cf. Bourgain 1992, 21. 
II 33 (p. 44, 38-45, 1 sq.) [Comes] camelos quattuor [...] Alexandro papae, qui tunc temporis uice beati Petri prudenter et catholice exsequebatur, apud Romam repraesentat.

Il est permis de supposer ici une omission, le verbe de la subordonnée relative, exsequebatur, ne pouvant se passer d'un complément d'objet explicite ${ }^{65}$. On suppléera à cette lacune en lisant uices, comme en III 39 (p. 81, 21 sq. : [Boamundus] patris uices haud grauiter exequebatur) ou en IV 7 (p. 89, 15 sq. : [...] absente episcopo, uices sibi delegatas cum omni prudentia et moderatione exequebatur $)^{66}$.

\section{37 (p. 47, 13 sq.) dux et comes Rogerius prius in prouincia Cusentii castrum qui-} dem Ragel expugnauerant et pro libitu ordinauerant.

$\|$ prius om. $B \|$ quidem $C Z$ : quod $B \| \operatorname{ragel} C M e C a$ : rauel $B$ regale $Z$, ed. pr. rogel Pontieri $\|$ expugnauerant... ordinauerant $C Z$ : -runt... -erunt $B$, Pontieri $\|$ et om. $B \|$

quidem ne semble avoir embarrassé personne, bien qu'il soit ici hors de sa place ${ }^{67}$. Tous les traducteurs ignorent d'ailleurs la nuance attachée ordinairement à cette

65. Exsequi n'est pas susceptible d'être employé absolument. Dans Sénèque, dial. 3, 12, 2, seul passage où les dictionnaires signalent un emploi absolu de ce verbe (cf. Benoist-Goelzer, p. $549^{\mathrm{A}}$, ou Gaffiot, p. $642^{\text {B }}$, s. v. 4), le complément d'objet se dégage aisément du contexte immédiat: Pater caedetur: defendam; caesus est: exequar, quia oportet, non quia dolet. Il n'y a aucun effet de sens lié à l'absence du complément non exprimé. Il reste que exequi intransitif est attesté une fois, en III 17 (p. 67, 1 sq. : Nostrae [...] naues, armamentis exportatis, minus erant ad exequendum paratae), avec, apparemment, la signification spéciale d'administrare pris absolument («faire la manœuvre»). On ne voit pas pourquoi les traducteurs modernes le prennent généralement comme synonyme d'insequi I persequi, les navires qu'il s'agit de «poursuivre» étant au mouillage, anchoris fixis (p. 66, 37-67, 1). Ici, les traducteurs, s'accommodant du texte imprimé, se sont partagés : tantôt ils font d'exsequebatur l'équivalent syntaxique et sémantique de fungebatur (cf. Avenel 2001, II, 109; Lo Curto 2002, 149), et tantôt, détachant le substantif du verbe, ils donnent à ce dernier la valeur - toute sallustéenne - d'un agitabat absolu (cf. Loud, ad loc.; Wolf 2005, 111).

66. Cf. en outre IV 7 (p. 90, 3: ad Mariae uices cum Martha exequendas transiit) et IV 16 (p. 94, 18 sq. : [...] Petrum Mortonensem, cui uices suas plurimum commiserat exequendas, [...] mittit). En III 40 (p. 82, 2 sq.) ad quod uenerat exequens, le complément direct d'exequens est représenté par la subordonnée relative, et l'antécédent $i d$ du relatif est omis ( «réalisant ce pour quoi il était venu»). Cette ellipse du complément direct est fréquente, quand c'est un pronom qu'il faut sous-entendre: cf. Desbordes 2005, 122 sq. Mais Malaterra omet également le pronom antécédent du relatif dans des constructions coordonnées dont le second constituant conjoint est une subordonnée relative: $\mathrm{cf}$. III 24 (p. 71, 18) timore eum et qui cum ipso abibant amittendi; III 37 (p. 79, 20 sq.) peditum copias et quae infirmiores erant ante se ponens; III 40 (p. 82,1) filium et quos cum ipso apud extremas partes dimiserat [...] ; IV 25 (p. 103, 25 sq.) rex episcopum Leocastrensem et qui cum ipso reuersuri uenerant aliquantisper secum commorari faciens; III 11 (p. 63, 38 sq.) Comes [...] urbem [...] militibus et quae his necessaria erant munit (ici, le iis quae de Pontieri sanctionne une faute imaginaire).

67. On ne voit pas en vue de quel effet de style la particule est insérée au cœur d'une construction appositionnelle. Dans la très grande majorité de ses emplois - vingt-cinq exemples sur quelque trente-sept occurrences -, quidem est en relation avec une particule restrictive (at, autem, sed surtout, tamen et uero). Ici, un quidem assévératif est tout simplement oiseux. 
particule. Mais K. B. Wolf, améliorant son texte source, traduit: « a certain fortress called Rogel ${ }^{68}$, comme s'il avait lu dans Pontieri : castrum quoddam quod Rogel dicitur. Cette innovation, que son auteur n'a pas motivée et qui n'a que très peu de chances de retrouver le texte authentique, a néanmoins le mérite de provoquer la critique et de suggérer une explication de la difficulté: quidem serait soit une interpolation soit le développement aberrant d'une forme abrégée. On sait que le digramme $q d$ surmonté d'un tilde peut se lire quidem ou quod dicitur. Faut-il dès lors rétablir castrum quod dicitur Ragel? Rien n'est moins sûr : quod dicitur n'est pas l'ordre usuel. Lorsque Malaterra recourt à une relative pour spécifier un toponyme, il insère invariablement le nom propre désignant le lieu entre le relatif et le verbe ${ }^{69}$. Il faut donc poser un état préalable du texte dans lequel l'ordre originel aura été perverti par une interversion ${ }^{70}$ et lire, en définitive, castrum quod Ragel dicitur. Mais mieux encore peut-être que cette faute indirecte, à trois degrés - une interversion crée une formule toute faite qui, une fois abrégée, prête à confusion ${ }^{71}-$, le contexte immédiat rend compte de la présence de quidem : à la suite d'un saut vertical de castrum (p. 47, 13) à castrum (p. 47, 14), un copiste se met en devoir de tracer quoddam. Seulement, s'étant rendu compte presque aussitôt de sa méprise, il s'interrompt avant

68. Cf. Wolf 2005, 115, appliquant à Rogliano les mots qui servent, une ligne plus loin, à présenter Aiello : « a certain fortress called Aiello ».

69. On en dénombre quarante-cinq exemples dans toute la chronique. La construction grammaticale du toponyme peut varier - le nominatif cède parfois la place au génitif; on relève également quelques formes locales, indéclinables -, mais jamais l'ordre des constituants de la subordonnée ne change : la place finale y est réservée au verbe. Quand une précision subsidiaire est introduite, elle s'insère de manière constante entre le relatif et l'attribut: cf. I 13 (p. 14, 27) quod corrupte ab incolis Montolium dicitur; II 16 (p. 34, 8) in ripa fluminis quod lingua eorum Guedetani dicitur. Cet ordonnancement de la subordonnée n'est pas modifié lorsqu'au passif dici est substituée une locution verbale équivalente: cf. III 24 (p. 72, 3 sq.) aliud castrum, quod ex nomine insulae Corofon nomen accepit, $[\ldots]$ suae mancipat seruituti. La seule relative de cette sorte qui fasse exception se lit en II 36 (p. 46, 30-32), mais la variante d'ordre se double d'une mutilation : in monte qui postea Tarentinus $<$ dictus est $>a b$ abundantia tarentarum a quibus ibidem exercitus eorum [sc. Roberti ducis et comitis] plurimum uexatus est. Dans la représentation schématique que nous pouvons tracer de toutes les autres relatives de même nature, l'attribut Tarentinus et le supplément que Pontieri a indiqué, dictus est, sont attendus après uexatus est - seule place propre à rendre compte, d'ailleurs, de la chute de ce supplément (saut du même au même amorcé par le retour de -tus est). La longueur du segment intermédiaire entre le pronom et son attribut et qui exprime le renseignement étiologique ne justifie pas, à soi seule, cette double rupture; cf. Desbordes 2005, 151, n. 114. De ces termes intimement liés, seul l'adjectif aura été rétabli - et incorporé au texte en fausse place. La correction suppose un lecteur assez bon connaisseur de la topographie pour spécifier une dénomination qui n'est attestée nulle part ailleurs (cf. Avenel 2001, IV, 529), mais peu au fait des habitudes de style du chroniqueur.

70. Une telle interversion est difficilement explicable. Son unicité suffit à lui dénier toute valeur critique.

71. Dans ce cas, la fausse leçon de $\mathrm{B}$, quod, représenterait la même forme abrégée, après déplacement du tilde : qđ. 
d'avoir écrit l'élément dans son entier. C'est ce quoddam mutilé - mais sous quelle forme? - que les copistes postérieurs auront, dans des circonstances qui demeurent obscures, mélu ou diversement interprété ${ }^{72}$. Selon cette hypothèse, quidem, provenu d'une anticipation, doit être éliminé comme intrus : dux et comes [...] castrum Ragel expugnauerant et pro libitu ordinauerant.

II 43 (p. 51, 1-4) Imperator [...] classem innumeram parare fecit, cui Gozelinum de Corintho - quendam natione Normannum et in palatio post imperatorem secundum paucis, quia strenuus armis et consilio callens erat-ducem praeponens, cum multis copiis Barensibus succurrendum disponit.

$\|$ innumeram om. $Z$, ed. pr. in mari Pontieri $\|$ gozelinum $C$ Ca, Avenel: goleninum $Z$ gocessinum $B$ gocelinum ed. pr. Pontieri $\|$ corintho $C \mathrm{Ca} M e$ : corticho $B$ orencho $Z$, edd. uett. $\|$ normannum $Z B$ : -ndum $C C a \|$ quia strenuus $C B$ : qui | a strenuis $Z \|$ praeponens $Z B$ : disponens $C \|$ barensibus $C Z$ : -ses $B \|$

Nous avons eu déjà l'occasion de dénoncer les non-sens de l'édition Pontieri en matière de ponctuation ${ }^{73}$. Ici, le savant italien a introduit une incidente dans la subordonnée relative, ouvrant une parenthèse pour indiquer l'origine du personnage, sa position à la cour et les qualités auxquelles il doit ce rang dans la hiérarchie. Mais outre que son point de départ peut être légitimement discuté, cette parenthèse inclut un élément qui lui est étranger. En effet, une analyse de tous les emplois de quidam dans la chronique révèle que le pronom indéfini se rattache en l'occurrence au nom propre qui le précède, non à l'ethnique qui le suit. Comparer par exemple I 7 (p. 10, 20) Maniacus autem quidam, natione Graecus; I 7 (p. 11, 12) Archadius

72. B, ou son modèle, a remanié la phrase pour pouvoir construire quod: castrum quod Rauel expugnauerunt pro libitu suo ordinauerunt. Du toponyme, il a fait un locatif et il a retranché la coordination qui reliait les deux propositions. Doit-on en conclure que quod est un vestige de quoddam? qđ, que l'auteur du saut du même au même, s'étant immédiatement corrigé, avait sans nul doute exponctué, aura été alors mésinterprété et développé en quidem (cf. C Z).

73. Cf. Desbordes 2002, 46 et n. 26; 2005, 137, n. 68 ; voir aussi supra, n. 23 et 33. À ce propos, la docilité des traducteurs est parfois déconcertante. Ici, paucis, isolé par une virgule de la fin de la parenthèse, a été rapporté au terme antécédent, secundum, bien que celui-ci soit déterminé déjà doublement (in palatio; post imperatorem) et d'une manière qui exclue un complément de cette sorte: post imperatorem secundus équivaut à «le premier après l'empereur ». Ainsi, par la faute d'une virgule illogique, le second personnage de l'État byzantin est subordonné à des pauci jouant auprès de l'empereur le rôle que Malaterra attribue expressément au Normand Gocelin : «dopo l'imperatore secondo a pochi nel palazzo " (Lo Curto 2002, 171); " a man of Norman stock who was considered second to very few men in the palace, after the emperor himself » (Wolf 2005, 121). Aucun de ces deux traducteurs n'a reconnu dans ce paucis l'ablatif du substantif indiquant qu'on exprimera « en peu de mots » ce qu'on veut dire. Paucis a déjà été vu en I 2 (p. 8, 11: procinctus terrae paucis perstrinximus). On en compte trois exemples dans le Catilina de Salluste (cf. Catil. 4, 3; 5, 9; 38, 3), quatre dans son Jugurtha (cf. Iug. 15, 1; 17, 1;95, 2;110,6). On ne conclura pas pour autant à un emprunt: l'expression est usuelle. 
quidam, qui urbi principabatur; I 8 (p. 11, 24) per Arduinum quendam, Italum ; I 22 (p. 36, 15) Nichel quidam, potentior castri; II 44 (p. 52, 4) Costo Pelagan quendam stratigotum statuerat; III 15 (p. 66, 11) Euiscardus quidam, natione Brito ; IV 7 (p. 89, 6) Gerlandum quendam, natione Allobrogum; IV 7 (p. 89, 9) Stephanum quendam, Rothomagensem ${ }^{74}$, honestae uitae uirum. En conséquence de quoi nous proposerions la ponctuation suivante: Imperator [...] classem innumeram parare fecit, cui Gozelinum de Corintho quendam, natione Normannum et in palatio post imperatorem secundum - paucis, quia strenuus armis et consilio callens erat-, ducem praeponens, cum multis copiis Barensibus succurrendum disponit.

La véritable difficulté de ce morceau de texte est ailleurs. Selon M.-A. Avenel, la leçon de C, disponens, serait une anticipation de disponit (p. 51, 4), au-dessus duquel praeponens se serait trouvé à un moment donné de la transmission ${ }^{75}$. C'est pourquoi, comme Pontieri, elle la relègue dans l'apparat. On ne saurait leur donner tort: il est impossible d'appuyer cette leçon de C par la confrontation de passages parallèles tirés de la chronique ou d'autres auteurs - ceux du moins dont les tours et expressions remarquables sont enregistrés dans les dictionnaires. Mais plus encore que cet effet de suggestion régressive, c'est l'interprétation que M.-A. Avenel donne de ducem qui rend praeponens nécessaire : «l'empereur [...] fait armer une flotte innombrable, qu'il place sous la direction du duc Gocelin de Corinthe - un Normand [...] ». Comme elle croit pouvoir y reconnaître un titre nobiliaire ${ }^{76}$, il faut un verbe exprimant en propre l'idée de commandement. Sans doute dux désigne-t-il, dans la très grande majorité des emplois de ce mot, un « duc»-quasi exclusivement normand, et cela dès l'évocation du héros fondateur, Rollon ${ }^{77}$-, mais il prend aussi, occasionnellement, l'acception de « commandant en chef d'une armée » ${ }^{78}$ ou celle d' "instigateur

74. Généralement mal ponctué, comme Italum en I 8 (p. 11, 24). Tous ces éléments de caractérisation peuvent commuter : potentior castri avec qui urbi principabatur; Italum avec natione Brito et Rothomagensem avec natione Allobrogum.

75. Cf. Avenel 2001, IV, 555. Contrefaute: I 7 (p. 11, 11 sq.) ciues ab urbe progressi $[\ldots]$ cum Maniacensibus congrediuntur (Pontieri, Avenel : progrediuntur $\mathrm{AC}$ om. ZB, edd. vett.). On ne saurait alléguer à l'appui de la lecture de C ce passage de III 40 (p. 81, 32 sq.) omnibus suis ordinatissime dispositis, plurimo exercitu uersus Graeciam quae coeperat executum accelerare disponit, qui réunit en deux exemples les constructions et les sens usuels de disponere. - Sur une répétition qui a tout l'air d'une négligence, cf. II 40 (p. 49, 30 sq.) Sicque ducem laesum credens, incasso uulnere spiculo amisso, nihil sibi fuga utilius credens, eqs.

76. C'est le titre que porte le personnage en question dans l'Anonyme du Vatican, 764: Gerolinus quidam, Normannigena, Dux Corinthiorum, quem Imperator nauibus in Apuliam ituris prefecerat (cité par M.-A. Avenel, comm. ad loc., n. 472). Dans sa lettre au gouverneur de Bari, « le duc de Corinthe salue le duc de Bari» : Gerolinus dux Corinthiorum Archerio duci Barensium salutem (Anon. VAT. 764).

77. Cf. I 1 (p. 7, 4) ; I 2 (p. 7, 15; 8, 8) ; I 40 (p. 25, 14).

78. Cf. duce exercitus (I 10 [p. 13, 15], à propos du catépan Bojoannès, déjà mentionné p. 13, 6 en qualité de "généralissime» : duce Anon [texte de M.-A. Avenel; la tradition est incertaine]); si ducem mutauerunt (II 41 [p. 50, 8], pour désigner le nouveau chef des Sicilienses coalisés contre le comte 
d'une entreprise ${ }^{79}$. Ici, comme nous y invitent d'ailleurs expressément les passages correspondants de l'Anonyme du Vatican, 764 (nauibus in Apuliam ituris prefecerat; cf. n. 71), et de Guillaume d'Apulie (III 117: [Gocelinus] nauibus his iussu praeponitur imperiali), $d u x$ vise le chef de la formation navale byzantine que l'empereur romain Diogène envoie à l'aide de Bari assiégé, celui que Roger fera prisonnier après l'abordage du vaisseau amiral (cf. II 43 [p. 51, 22-27]). D'autre part on ne voit pas la raison qui aurait rendu nécessaire le rejet de cette détermination par le nom commun loin du groupe du nom propre, dont, antéposé ou postposé, il n'est ailleurs jamais séparé. Seulement cette modification du sens de ducem soulève une difficulté nouvelle: le substantif fait double emploi avec praeponens ${ }^{80}$. C'est pourquoi nous gageons - sans repousser l'explication suggérée par M.-A. Avenel - que l'hyparchétype commun portait déjà disponens, qu'un ancêtre de $\mathrm{ZB}$ aura arrangé en praeponens sous la suggestion du contexte. Nous supposons que la substitution déclenchée par disponit a entraîné une déformation du préverbe et proposons soit imponens ${ }^{81}$ soit apponens $^{82}$, voire le simple ponens ${ }^{83}$.

Roger); qui dux hostium erat (II 43 [p. 51, 22], à propos de Gocelin lui-même) et per suum ducem, nomine Aliuum (IV 25 [p. 103, 5 sq.], glosé "generale di Colomano, re d'Ungheria " par Pontieri 1928, 124).

79. Cf. duce Nichel (II 22 [p. 36, 20], l'inspirateur du complot visant à assassiner Betumen).

80. praeponens ne devient "préférable pour le sens » qu'à la condition que ducem soit la traduction latine du nom grec de la dignité ( «megas droungarios»? «megas dux»?) conférée à Gocelin lorsque l'empereur lui confia le commandement de la flotte byzantine basée à Durazzo : «l'empereur fait armer une flotte considérable, au commandement de laquelle il porte, avec le titre de $d u x$, un certain Gocelin de Corinthe, un Normand [...]». Comparer III 30 (p. 75, 23 sq.) [...] quem comes apud Cathaniam maiorem urbi praefecerat; IV 7 (p. 89, 5-7) Huic ecclesiae Gerlandum quendam, [...], episcopum ordinans, praefecit; Svlp. SEv., chron. 1, 53, 5 (p. 54, 21 Halm) Eisdemque [sc. la turba inutilis que le vainqueur assyrien a épargnée] rex Sedechias praepositus ; 2, 26, 6 (p. 81, 27-82, 1 Halm) Imposito Iudaeis stipendio, procuratorem eis Antipatrum quendam, Ascalonitem, praeposuit [sc. Pompeius]. Sur des termes grecs désignant une fonction administrative translittérés en caractères latins, cf. I 32 (p. 22, 26 sq.) Giracii praesopus - quem nos praepositum dicimus; II 44 (p. 52, 4 ; 11 ; $22 ; 23 ; 24)$ stratigotus, et voir Avenel 2001, III, 414; IV, 563.

81. Sur imponere «i. q. praeficere, praeponere, collocare», cf. TLL VII 2, 655, 80-656, 44 (J.B. Hofmann, 1938). Par une coïncidence curieuse, dans Амм. 25, 1, 8, là où le plus ancien des mss conservés donne imposuit, Ernesti et, à sa suite, les éditeurs modernes lisent disposuit.

82. Cf. Амм. 21, 8, 1; 22, 7, 7; 25, 1, 8; 29, 5, 35 .

83. Il y a dans la Bible de nombreux exemples de cet emploi de ponere: $\mathrm{cf} .1 R 16,2 ; 20,24 ; 2 R 23,5 ; 1$ Ch 26, 10 ; 2 Ch 9, 8; 2 Esd 13, 26; etc. Cf. encore Greg. Tvr., Franc. 8, 41 (p. 218, 24-26 KruschBuchner) : Fredegundis uero Melantium, quem prius episcopum posuerat, ecclesiae instituit. Sur "ponere pro imponere", cf. Norberg 1939, 177 sq. - D'autres verbes sont évidemment possibles: cf. TLL V 2327, 3-26 DVX (IVNCTVRAE) (O. Hey, 1934). Nous n'avons retenu que les formes unies à disponens par une communauté de radical. 


\section{44 (p. 52, 4 sq.) nimia insolentia defluens}

insolentia defluens est une curiosité d'expression que les traducteurs se sont dispensés de rendre très exactement ${ }^{84}$, bien que, donnée par tous les manuscrits, elle ait été adoptée par tous les éditeurs. Il faut reconnaître qu'elle ne fournit aucun sens acceptable et qu'il y a lieu à correction ${ }^{85}$. Un changement de suffixe, par substitution de i à e ${ }^{86}$, y pourvoira : diffluens, ainsi que l'enseigne le Mittellateinisches Wörterbuch, III 4, p. 617, 51 sq. (Staub, 2002), apparait comme substitut de luxurians, et il désigne ici les déportements d'un gouverneur que son arrogance extrême pousse à toutes les exactions: «Le duc avait institué stratigot un certain Costo Pelaga qui, bouffi d'orgueil, se portait à tous les excès, accablant d'outrages tous les gens honorables, les femmes comme les hommes, sans épargner même sa parentèle ${ }^{87}$.

\section{44 (p. 52, 30) Istud hic inseruimus eqs.}

$\|$ hic $Z B$ : hoc $C \|$ inseruimus $C M e$, Avenel: in seruitium $B$ inscribimus $Z$, ed. pr. Pontieri $\|$

Inserere construisant son second complément au datif ${ }^{88}$, il est permis de se demander si le hoc que C oppose à la vulgate imprimée, hic, tiré de ZB, et dont Pontieri

84. Lo Curto 2002, 175 ( «con grande arroganza»), et Wolf 2005, 123 ( "with great insolence»), ne voient dans defluens qu'une cheville. M.-A. Avenel lui prête un sens et une construction qui ne sont pas autrement attestés : "se laissant aller aux pires insolences" (cf. Avenel 2001, II 131). On ne saurait non plus donner à defluere le sens d'abundare ou d'affluere (cf. TLL V 1,365, 33 sq. [G. Jachmann, 1910]), l'idée d' " abondance » étant déjà exprimée par l'épithète nimia.

85. Sauf à considérer que nous avons affaire ici à un nominatif absolu (= « son orgueil démesuré se donnant carrière »?). Mais les emplois d'insolentia et de defluere ne plaident guère en faveur de cette construction. Le verbe est appliqué une fois à un froid «de mort» (pruina mortalitatis [...] defluens: I 27 [p. 21, 15] - par imitation de la mors defluens de Si 51, 13?); ailleurs, il est utilisé exclusivement dans son sens propre (cf. I 1 [p. 7, 7] quo Secana fluuius in mare defluens intrat; III 24 [p. 71, 36 sq.] ubi fluuius Bayosae in mare defluit; IV 22 [p. 100, 30] super fluuium qui a Sancto Marco defluit). Quant à insolentia, il forme, sur le modèle de in iram prorumpere ("éclater de colère " [cf. IV 21 (p. 99, 36)]), une expression pittoresque, in insolentiam prorumpere, pour qualifier l'attitude de ceux qui, étant entrés en rébellion, «donnent libre cours à leur arrogance » (quatre occurrences: IV 9 [p. 90, 31] ; IV 18 [p. 98, 10 sq.]; IV 21 [p. 99, 18] et IV 26 [p. 105, 7]). Le dernier emploi qui mérite attention n'ouvre pas davantage la voie au nominatif absolu: Guillelmus de Grantemanil [...], in insolentiam procliuis declinans, Rossanam [...] peruadens intrat (IV 21, p. 99, 19-21). L'analyse de tous ces morceaux de texte, dans lesquels le substantif occupe la position subalterne de complément, invite en II 44 à faire de nimia insolentia un instrumental, non le sujet d'un participe absolu.

86. Voir Desbordes 2005, 127 sq., n. 39; 132. La confusion des deux formes n'est pas l'apanage des copistes de la chronique. Elle se rencontre par exemple dans des recensions défectueuses de Cicéron, de orat. 3, 131, de Macrobe, Sat. 2, 4, 22 (diffluens vulgo: defluens codd. [p. 146, 24 Willis]), ou des Carmina Burana (63, refl. 6 : difluere alterne avec defluere). "Persaepe diffluere et defluere permutantur» (TLL V 1104, 43 sq. [J. Rubenbauer, 1912]).

87. Dux Costo Pelagan quendam stratigotum statuerat, qui, nimia insolentia diffluens, quosque ingenuos, uiros uel mulieres, nec etiam suae consanguinitati parcens, contumeliis afficiebat.

88. Cf. I 11 (p. 14, 7 sq. : Quia perlongum est huic operi [...] inserere qualiter in Apulia egerint); III 41 (p. 82, 6 sq. : Mirabile quoddam praesagium [...] huic operi inserere dignum nobis uisum est); IV 18 
fait si peu de cas qu'on n'en trouve pas trace dans son apparat, ne représente pas l'adverbe huc. Le choix est malaisé : l'adverbe n'est pour ainsi dire pas attesté dans la chronique ${ }^{89}$; d'autre part, insero admet parfois l'ablatif à côté de l'accusatif d'objet: cf. PLin., nat. 3, 65 Non alienum uidetur inserere hoc loco exemplum religionis antiquae; Hier., epist. 20, 1 in hoc loco non esse nomen insertum. Hic se retrouve d'ailleurs à deux reprises dans des emplois analogues: cf. III 42 (p. 82, 30: finis [...] huius libri hic ponendus est) et IV 29 (p. 107, 20: cuius sententiam hic subtitula$m u s)^{90}$. ZB donne donc ici un texte satisfaisant, sans néanmoins parvenir à éloigner toute suspicion.

Olivier Desbordes

Université de Caen Basse-Normandie

\section{Références bibliographiques}

Boetticher G. (1830), Lexicon Taciteum sive de stilo C. Cornelii Taciti, Berlin, G. C. Nauck.

Bourgain P. (1992), "Sur l'édition des textes littéraires latins médiévaux », Bibliothèque de l'École des chartes, 150, p. 5-49.

D’Angelo E. (2003), «Un “doppio” prologo al “De rebus gestis Roberti et Rogerii” di Goffredo Malaterra? », in Storiografi e cronologi latini del Mezzogiorno normanno-svevo, Naples, Liguori Editore (Nuovo Medioevo ; 69), p. 134-142.

Desbordes O. (2002), "Notes de critique textuelle sur le livre premier de Geoffroi Malaterra», in Mélanges Pierre Bouet. Recueil d'études en hommage à Pierre Bouet, F. Neveux, C. Bougy (éd.), Caen, Annales de Normandie (Cahier des Annales de Normandie; 32), p. 39-47.

(p. 97, 7 sq. : ordo temporis damnosum dolorem [...] nos quin huic libro inseramus praetergredi uetat). Comparer Liv. 5, 21, 8 Inseritur huic loco fabula; VAl. MAX. 1, 6 ext. 1; 3, 2, 17; 3, 8, 6; Svet., Dom. 18, 2 ; MACR., Sat. 3, 9, 4 .

89. Huc se lit seulement en III 18 (v. 3) certamen durat, comes huc persistere curat - incorrectement construit avec un verbe de repos; mais illuc, bien mieux représenté que lui, présente les mêmes irrégularités: cf. II 16 (p. 34, 2: per octo dies illuc morati sunt); II 19 (p. 35, 15 sq. : illuc sollemnes nuptias celebrauit); III 36 (p. 78, 35 : [...] patris thesauros, qui illuc seruabantur).

90. Ailleurs, hic est exposé aux mêmes aléas de construction que huc ou illuc: il se rencontre auprès de verbes qui, en règle générale, exigent un accusatif indiquant la direction - comme si le chroniqueur n'envisageait que le terme du mouvement. Cf. IV 22 (p. 100, 32) Hic Guillelmus [...] accessit; IV 25 (p. 103, 16 sq.) Hic Vincurius [...] missus cum quinque milibus armatorum obuius fuit. Sur certaines confusions dans l'emploi de $u b i$ et quo, cf. Desbordes 2005, 143 sq. et n. 86. La construction des compléments de lieu mériterait une étude spéciale. 
Desbordes O. (2005), « Nouvelles notes critiques sur les deux premiers livres de la chronique de Geoffroi Malaterra », Kentron, 21, p. 111-159.

Flobert P. (1975), Les Verbes déponents latins des origines à Charlemagne, Paris, Société d'édition «Les Belles Lettres» (Publications de la Sorbonne, série «NS Recherches »; 17).

Geoffrey Malaterra (Wolf 2005), The Deeds of Count Roger of Calabria and Sicily and of his Brother Duke Robert Guiscard, K. B. Wolf (trad.), Ann Arbor, The University of Michigan Press.

Geoffrey of Malaterra (Loud), The Deeds Done by Count Roger of Calabria and Sicily and of Duke Robert his Brother, G.A. Loud (trad.), Leeds, University of Leeds, School of History (Medieval Texts in Translation. The Norman Kingdom of Sicily) (http:// www.leeds.ac.uk/history/weblearning/MedievalHistoryTextCentre/medievalTexts.htm).

Geoffroi Malaterra (Pontieri 1927-1928), De rebus gestis Rogerii Calabriae et Siciliae Comitis et Roberti Guiscardi Ducis fratris eius auctore Gaufredo Malaterra monacho Benedictino, E. Pontieri (éd.), Bologne, N. Zanichelli (Rerum Italicarum Scriptores, Raccolta degli storici italiani dal cinquecento al millecinquecento ordinata da L.A. Muratori, t. V-1, fasc. $1 ; 2-3)$.

Goffredo Malaterra (Lo Curto 2002), Ruggero I e Roberto il Guiscardo, V. Lo Curto (éd. et trad.), Cassino, Francesco Ciolfi Editore.

Hand F. (1829; 1832), TVRSELLINVs seu de particulis Latinis commentarii, Leipzig, Weidmann, Volumen primum (1829), Volumen secundum (1832).

Havet L. (1911), Manuel de critique verbale appliquée aux textes latins, Paris, Librairie Hachette (= Rome, «L'Erma» di Bretschneider, 1967).

Hofmann J.B., Szantyr A. (1965), Lateinische Syntax und Stilistik, Munich, C.H. Beck'sche Verlagsbuchhandlung (Handbuch der Altertumswissenschaft; II. 2. 2).

JAnson T. (1979), Mechanisms of Language Change in Latin, Stockholm, Almqvist \& Wiksell International (Acta Universitatis Stockholmiensis. Studia Latina Stockholmiensia ; 23).

Krebs J.P., Schmalz J.H. (1905), Antibarbarus der lateinischen Sprache, $7{ }^{\mathrm{e}}$ éd., Bâle - Stuttgart, Benno Schwabe \& Co, 2 tomes (réimpr. Darmstadt, Wissenschaftliche Buchgesellschaft, 1984).

Kühner R., Stegmann C., Thierfelder A. (Kühner et al. 1976), Ausführliche Grammatik der lateinischen Sprache, $5^{\mathrm{e}}$ éd., Zweiter Teil : Satzlehre, Darmstadt, Wissenschaftliche Buchgesellschaft, 2 vol.

Lavarenne M. (1933), Étude sur la langue du poète Prudence, Paris, Société française d'imprimerie et de librairie - Ancienne Librairie Lecène, Oudin \& $\mathrm{C}^{\mathrm{ie}}$.

Lucas-Avenel M.-A. (Avenel 2001), Édition critique avec traduction et commentaire des deux premiers livres de la chronique de Geoffroi Malaterra, thèse de doctorat de Langues et littératures anciennes sous la direction de L. Callebat et de S. Fodale, Université de Caen Basse-Normandie, 4 fasc. (I : 1-148 p.; II : 149-290 p., III : 291-430 p.; IV : 431-603 p.; I-VI: résumé italien ; 9 hors-texte; table des matières) (dactyl.). 
Marouzeau J. (1962), Traité de stylistique latine, 4 éd., Paris, Société d'édition «Les Belles Lettres» (Collection d'études latines publiées par la Société des études latines, série scientifique; 12).

Moussy C. (1966), Gratia et sa famille, Paris, Presses universitaires de France (Publications de la Faculté des Lettres et Sciences humaines de l'université de Clermont-Ferrand, $2^{\mathrm{e}}$ série; XXV).

Norberg D. (1939), In Registrum Gregorii Magni studia critica II, Uppsala - Leipzig, A. B. Lundequistska Bokhandeln - Otto Harrassowitz (Uppsala Universitets Årsskrift [Recueil de travaux publié par l'université d'Uppsala] ; 1939, 7).

Ogilvie R.M., Richmond I.A. (1967), Cornelii Taciti De Vita Agricolae, Oxford, Clarendon Press.

Resta G. (1964), «Per il testo di Malaterra e di altre cronache meridionali », in Studi per il CL anno del Liceo-Ginnasio «T. Campanella» (1814-1964), Reggio di Calabria, p. 3-6o.

Saint-Denis E. de (1935), Le Vocabulaire des manœuvres nautiques en latin, Mâcon, Protat Frères.

Sтотz P. (1998), Handbuch zur lateinischen Sprache des Mittelalters. Vierter Band: Formenlehre, Syntax und Stilistik, Munich, C.H. Beck'sche Verlagsbuchhandlung (Handbuch der Altertumswissenschaft ; II. 5. 4). 